\title{
La educación ambiental, una estrategia pedagógica para la conservación del humedal del barrio San Luis de la Ciudad de Florencia.
}

María Elena Bocanegra Arias Ruth Marina Calderón B.

Félix Humberto Cañón A.

Colegio Básico San Luis

Florencia - Caquetá

\section{Antecedentes}

El Colegio Básico San Luis inicia el trabajo sobre Educación Ambiental en 1997 amparado en la Constitución Nacional del 91, la Ley general 115 del 94 y el Decreto 1749 del 96. Teniendo en cuenta el contexto, la ubicación y la problemática cultural, económica, política y social de la Comunidad Educativa del barrio San Luis, nos dimos a la tarea de identificar los problemas en orden de prioridad para, desde lo pedagógico, encontrar una respuesta a éstos.

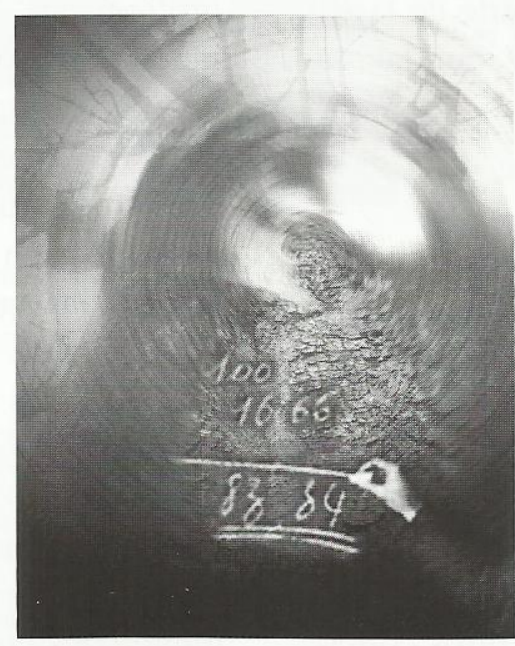

Conscientes de la necesidad de identificar estrategias para reducir la contaminación por residuos sólidos y aguas servidas del humedal del barrio San Luis de la ciudad de Florencia, así como de establecer una conducta ciudadana para conservar y manejar adecuadamente esta área como ecosistema estratégico y lograr cambios de actitud que conduzcan a establecer una relación bilateral y equitativa con el entorno, se formuló el proyecto La Educación Ambiental, una Alternativa Pedagógica para la Conservación del Humedal del barrio San Luis de la Ciudad de Florencia, cuyo reto es modificar el estilo de vida, integrando una comunidad activa y consciente, para hacer viable y operativa la transformación hacia una sociedad que aproveche racionalmente su entorno y brinde a las próximas generaciones un lugar habitable, armonioso y digno. 
El municipio de Florencia tiene una gran riqueza hídrica y de ecosistemas estratégicos como los humedales. El territorio donde fue fundada la ciudad de Florencia, era de humedales ubicados especialmente donde hoy es la bomba El Chorro y la Empresa de Licores del Caquetá, en el barrio la Consolata y el Humedal ubicado sobre la Avenida los Fundadores, hoy convertido en una alcantarilla que vierte sus aguas al Río Hacha.

El Humedal del barrio San Luis, aledaño al Colegio Básico San Luis, aparte de recibir la contaminación por aguas residuales, se ha convertido en foco de contaminación por el deposito de residuos sólidos arrojados por los habitantes de los barrios circunvecinos, generando pérdida de la biodiversidad de flora y fauna, proliferación de insectos y roedores, presencia de malos olores y degradación del paisaje, entre otros. Esto afecta la salud y calidad de vida de los habitantes ubicados en el área de influencia del Humedal, el cual se encuentra ubicado en una de las zonas más bajas de la ciudad de Florencia, a 240 metros sobre el nivel del mar, lo que significa que forma parte de las zonas consideradas de alto riesgo por inundaciones en la ciudad de Florencia.

\section{Humedales}

En la Convención realizada el 2 de febrero de 1971, en la ciudad de Iraní de Ramsar, sobre Humedales, donde participaron 96 países, los científicos concluyeron que los humedales son los "Riñones del Planeta" por su función de filtro de las aguas que pasan a través de ellos liberándolas de sedimentos, productos químicos y otros contaminantes. En esta Convención firmaron un tratado internacional para promover la conservación de los humedales y el uso sostenible de los mismos e igualmente institucionalizar, en esta fecha, el día internacional de los humedales.

De otro lado, la captación de sedimentos realizada por los humedales temporales cumple una función adicional en cadenas biológicas, al iniciar un proceso de sucesión vegetal.
En cuanto a su papel en el proceso de definición del paisaje, existen varias funciones que merecen atención. En primer lugar, el control de flujo y de picos de inundación: los humedales capturan y liberan lentamente el exceso de agua Iluvia y escorrentía, actuando a la manera de esponja de amortiguación; de la misma manera, la parte del ciclo hidrológico que se lleva a cabo en los humedales, sumado a los ciclos de nutrientes y materia y al flujo de energía de sus redes tróficas contribuyen a la regulación del fenómeno meteorológico tales como la precipitación y la fluctuación de la temperatura estabilizando el clima local y regional.

El territorio colombiano se encuentra bañado por 15 000 kilómetros de ríos, 3 millones de hectáreas en aguas superficiales, costas sobre los dos océanos y el $57 \%$ de páramos son surtidores de agua.

La importancia de los humedales radica en la estabilidad climática y en el control de inundaciones causadas por el invierno y de las sequías prolongadas. El exceso de lluvias fluye hacia zonas inundables, ciénagas y sistemas interconectados de cuerpos de agua, para evitar el desbordamiento de los ríos, y nivelar el volumen del agua de los acuíferos (agua subterránea contenida en capas geológicas en cantidades suficientes), Al mismo tiempo, las aguas Iluvias son absorbidas por humedales, suelos, bosques y almacenadas en reservorios naturales y corrientes subterráneas que afloran a la superficie en épocas de verano.

Además de la regulación hídrica, la estabilidad de microclima y del control de la erosión, "los humedales cumplen una función ecológica como es servir de dispensadores de nutrientes para plantas y animales y lógicamente como sustento para muchas personas que viven de la pesca"1.

Según especialistas que participaron en el Primer Taller Nacional de Humedales, organizado en Bogotá en 1991, opinan que el agua estacional es

Ministerio del Medio Ambiente. OIMT CEUDES. 1998. p. 70-75. 
más propicia para la generación de vida que el agua en movimiento y por eso los Humedales figuran entre los ecosistemas más productivos; son fuentes generadoras de alimento, cuna de reproducción y refugio de migración de numerosas especies de peces, animales terrestres y especialmente de aves.

La gran biodiversidad de especies que alojan y atraen los humedales, hace que este lugar dinámico se convierta en hoteles exclusivos para aves migratorias con vistosos colores, quienes realizan largos vuelos en diferentes épocas del año, pues no tienen hábitat propio.

Científicos colombianos clasifican los humedales de nuestro país de acuerdo al gradiente de altitud, en las siguientes zonas:

- Alto Andina o de Páramo: turberas, pantanos e innumerables lagunas.

- Andina: lagos y embalses.

- Tierras bajas: lagos, ciénagas, pantanos, madre viejas, meandros, sabanas inundables, bosques inundables, éstos son propios de la región amazónica.

- Costas: estuarios, manglares, catival, en este grupo se incluyen los sectores Ilamados costas abiertas.

El Departamento del Caquetá posee una gran riqueza de ecosistemas estratégicos; entre los más importantes tenemos: El Humedal de Guacochará, Humedal de Cartagena del Chairá, La Cocha y el Humedal del barrio San Luis, entre otros.

\section{El Humedal del barrio San Luis}

El humedal objeto de esta investigación y eje transversal de nuestro currículo, pertenece al grupo de tierras bajas, específicamente a los meandros. Su origen se debe al cambio de cauce del Río Hacha, como consecuencia de una inundación en 1965, dejando como resultado la desaparición de algunos barrios y de un gran número de personas que estaban asentadas en su margen. Posteriormente aparecen barrios como el Juan XXIII, el Versalles, Obrero y San Luis, entre otros.

Reconociendo la importancia de los humedales y concretamente el del barrio San Luis, se hace necesario el diseño de un plan de estudios que nos lleve a la recuperación y conservación del Humedal desde lo pedagógico, creando el PRAE del Colegio Básico San Luis que permee las diferentes áreas del conocimiento cuyo eje transversal sea el Humedal.

De modo que maestros y directivos del Colegio, realizamos un diagnóstico en cinco aspectos fundamentales que nos permitiera acercarnos la realidad: lo social, económico, cultural, político y comunitario y educativo. Trabajamos colectivamente en el diseño de una encuesta que se aplicó en la comunidad y que nos indicó que los principales problemas eran la contaminación ambiental, la baja autoestima y la violencia intrafamiliar. Concertadamente elegimos trabajar sobre la contaminación ambiental de nuestro entorno sin descuidar los otros problemas identificados.

Entendido el problema de la contaminación, se inició el proceso de investigación para identificar sus causas y consecuencias. Se realizó la actividad de reconocimiento del barrio y sus alrededores, encontrando que la "chuquía" (como se le llamaba) no era más que una caneca de basuras y el recipiente de aguas residuales. Para ello se hizo necesario identificar estrategias pedagógicas que nos llevaran a solucionar la problemática. Organizamos una comunidad académica de la institución con docentes de las dos jornadas y directivos del Colegio interesados en hacer una recopilación bibliográfica sobre humedales, problemas de contaminación, educación ambiental, procesos de investigación, modelos pedagógicos, referentes teóricos, sistematización de información entre otros. Se inicia el proceso de auto-capacitación conjuntamente con el Concejo Directivo, el Comité Técnico Ambiental y el Club de Amigos del Humedal organismos creados para fortalecer el proceso investigativo que íbamos a iniciar sobre la recuperación y conservación del humedal barrio San Luis. 
Después de realizar esta auto-capacitación iniciamos la construcción tanto de la visión y misión de la institución como la de un currículo y plan de estudios pertinente que optara en la solución de problemas y necesidades de la comunidad educativa. Para este fin agrupamos las nueve áreas obligatorias más las optativas en cuatro grandes áreas del saber:

1. Sociocomunicativa: Pertenecen a esta área las sociales, humanidades, ética, ciencias políticas. En esta área se busca a partir del contexto del humedal investigar sobre los universos culturales de nuestra comunidad educativa para recuperar sus costumbres e identidad.

2. Lógica matemática: A partir del entorno del humedal los estudiantes resuelven pequeños problemas en los cuales se aplican algunas temáticas de esta área como: extensión del humedal, estadística del área construida, clasificación de especies en conjuntos, elaboración de proyectos para reubicación de la gente que vive en las riveras del humedal.

\section{Ciencias naturales, tecnología y ambiente: Los} estudiantes realizan estudios taxonómicos de la flora y la fauna, del grado de contaminación del humedal, clasificación y recuperación de las especies en extinción, trabajo de proyectos en cerca viva.

4. Lúdica recreativa y deporte: Elaboración de inventario de juegos tradicionales y deportes de la comunidad. Creación de la semana ambiental pedagógica y creativa en la cual se realizan actividades como: maratón alrededor del humedal, conversatorio pedagógico ambiental entre instituciones educativas y no gubernamentales sobre innovaciones, proyectos o investigaciones ambientales propios de la región, exposiciones artísticas de los trabajos realizados por los estudiantes y presentación por parte de los docentes de los resultados obtenidos en cada uno de los proyectos investigativos de aula. Desde la Educación Artística, se crearon los símbolos. Entre ellos la bandera con los colores blanco, verde y su El libro viajero. Los estudiantes entregan el libro a cada familia para que respondan a unas encuestas y plasmen en él sus intereses, experiencias, expectativas y compromisos con el proyecto. Se realizó también el levantamiento de mapas, la elaboración de la maqueta, la creación de textos literarios como: acrósticos, fábulas, poesías, coplas y el rescate de los valores éticos en torno al Humedal.

Se organizan las áreas en estos cuatro grupos con el fin de formular estrategias pedagógicas que nos permitieran la articulación de contenidos y actividades con el eje temático central, el Humedal. Después de hacer una reflexión crítica de nuestro quehacer pedagógico, nos encontramos con la necesidad de cambio, por tal razón decidimos adoptar nuevas tendencias pedagógicas para aplicarlas en el proceso. Los paradigmas humanista, socio cultural y constructivista son las bases que nos permiten soportar el sistema de acciones que, a la postre, se proyectarán a la comunidad.

Una vez concertados los paradigmas, los contenidos pertinentes a cada grado y sus respectivas áreas, se diseñó el Plan de Estudios de Educación Ambiental, con tres niveles basados en los lineamientos curriculares para la educación ambiental emanados del Ministerio de Educación Nacional. (Ver recuadro en la pág. siguiente)

Cada nivel tiene su respectiva temática, el proceso de pensamiento y acciones proyectadas a la comunidad a través de subproyectos que alimenten el macroproyecto.

En el área de ciencias naturales se hace énfasis y se motiva la investigación tomando el Humedal como un laboratorio, espacio que permite hacer una clasificación taxonómica de fauna y flora de un gran número de especies animales y vegetales clasificándolos parcialmente. Además, se elaboró un mini vivero de plantas medicinales.

\section{Proceso de investigación}

Desde el área de Ciencias Naturales y la Educación ambiental, se inició un proceso teniendo en cuenta el Plan de Estudios de los grados $6^{\circ}$ a $9^{\circ}$. Específicamente la investigación se centra en taxonomía, suelos y los universos culturales de la comunidad. 


\section{ESTRUCTURA DEL PLAN DE ESTUDIO EN EDUCACIÓN AMBIENTAL}

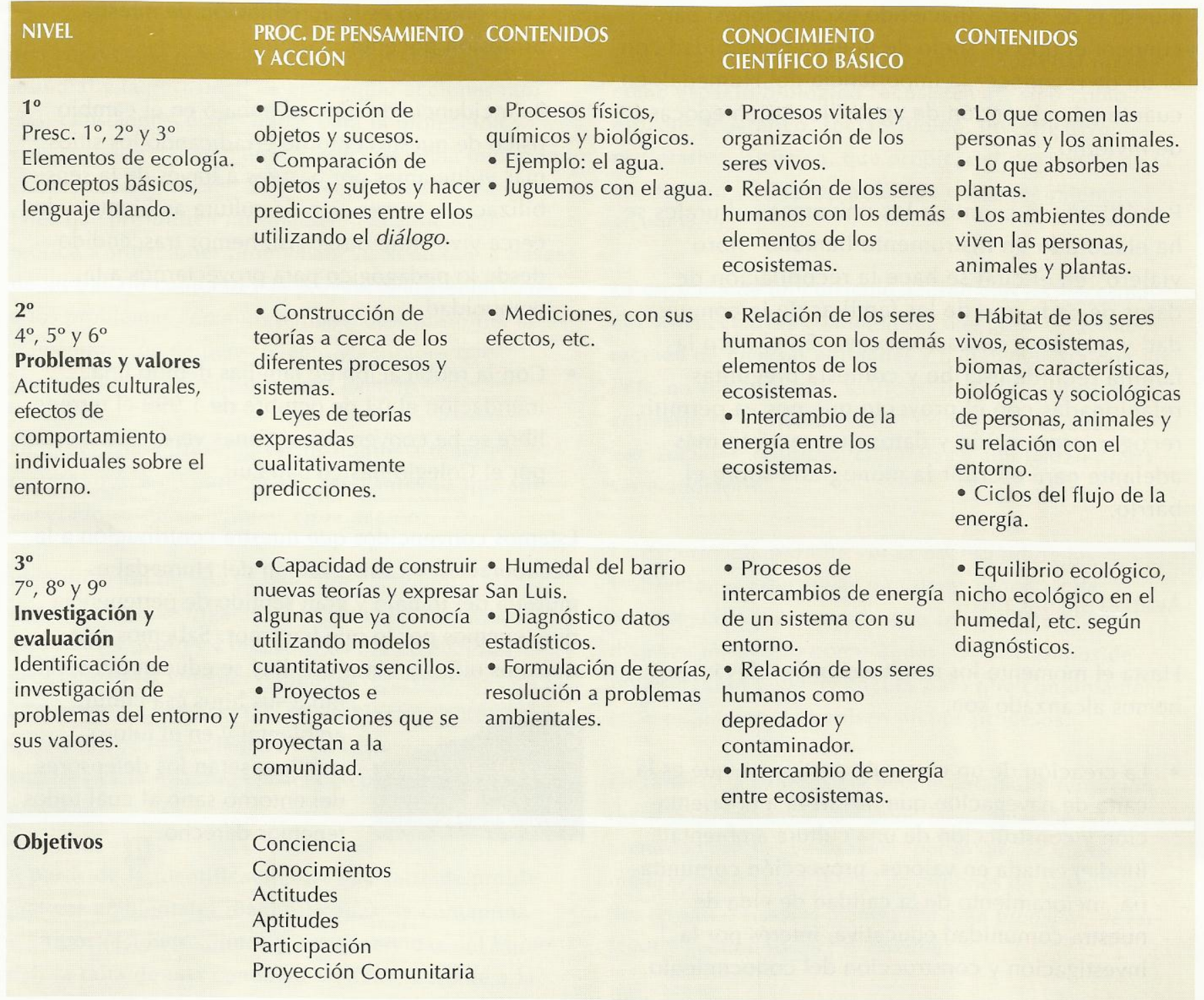

En taxonomía se han clasificado alrededor de 60 especies de plantas que existen en el humedal y se encuentran en peligro de extinción. Estas plantas se categorizaron por su raíz, tallo, flor, hojas, frutos y utilidad medicinal.

Estas plantas se les conoce con sus nombres comunes, nombre científicos, por familias y su utilidad. Se ha construido un minivivero con plantas y sus respectivos herbarios.

En cuanto a la clasificación de animales lo hemos hecho en dos grupos: Los insectos que son una gran variedad y los vertebrados. Para su clasificación se han construido insectarios, y álbumes con fotografías de animales propios del humedal con su respectiva taxonomía.

El Humedal se ha convertido en un laboratorio donde se ha realizado el trabajo de clasificación de la fauna propia del medio. Los animales encontrados se han clasificado en: vertebrados e invertebrados, la clase que agrupa a los animales según su reproducción, el orden, que agrupa a los animales según su alimentación y así sucesivamente, cada animal se clasifica teniendo en cuenta los diferentes taxones hasta conocer la familia, el género, y por último la especie. 
En cuanto a los suelos se ha investigado tomando muestras de tierra, (haciendo excavaciones) para conocer el tipo de suelo de nuestra comunidad con el fin de reconocer la importancia del Humedal, en cuanto a la absorción de aguas Iluvias en épocas de invierno.

Por último, en cuanto a los universos culturales se ha elaborado un instrumento Ilamado "libro viajero" en el cual se hace la recopilación de datos de cada uno de las familias de la comunidad y que pasa de casa en casa. En el libro la familia reunida escribe y contesta preguntas relacionadas con él proyecto que nos va permitir recoger información y datos que servirán más adelante para escribir la monografía sobre el barrio.

\section{Avances significativos}

Hasta el momento los resultados más relevantes que hemos alcanzado son:

- La creación de un currículo pertinente que es la carta de navegación que nos lleva a la orientación y construcción de una cultura ambiental fundamentada en valores, proyección comunitaria, mejoramiento de la calidad de vida de nuestra comunidad educativa, interés por la investigación y construcción del conocimiento.

- La participación en la red latinoamericana de formadores ambientales.

- La inclusión dentro del Plan de Ordenamiento Territorial en el Municipio de Florencia.

- El establecimiento de relaciones interinstitucionales con CORPOAMAZONÍA, EDAMAZ, la universidad de la Amazonía, el Instituto Sinchi, Policía Ambiental, Ministerio de Educación Nacional, Alcaldía de Florencia, Unidad Pedagógica, escuelas y colegios de la ciudad.

- La consolidación de una Comunidad Académica con un alto grado de pertinencia, comprometida con los avances tecnológicos de la educación y cuyo objetivo es la acreditación de nuestra profesión docente.

- La incidencia de nuestro trabajo en el cambio físico de nuestro entorno erradicando los sitios más vulnerables por basuras a través de la sensibilización, la creación de cultura ambiental y la cerca viva entre otros. Así, hemos trascendido desde lo pedagógico para proyectarnos a la comunidad.

- Con la reubicación de familias debido una inundación el 04 de octubre de 1.999 el terreno libre se ha convertido en zonas verdes manejadas por el Colegio Básico San Luis.

Estamos convencidos que nuestra contribución a la Recuperación y Conservación del Humedal es muestra del trabajo y gran sentido de pertenencia que tenemos por lo que hacemos. Sabemos que nuestros niños y niñas que hoy se educan transmiti-

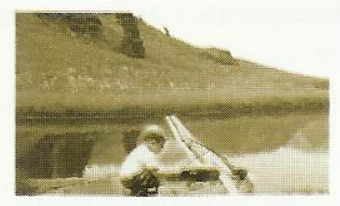
rán a sus hijos esa cultura ambiental y en el futuro próximo serán los defensores del entorno sano al cual todos tenemos derecho. 
Teniendo como referente la situación ambiental mundial y la necesidad de emprender acciones rápidas, conscientes y adecuadas para la protección y conservación del ambiente, resulta de suma importancia la implementación de proyectos escolares ambientales que posibiliten no solamente la aplicación teórica a situaciones problemáticas, si no que a través de un trabajo conjunto y la proyección de soluciones a los problemas cercanos e inmediatos posibilite la transformación de pensamiento y actitudes que generen un compromiso para el cambio.

La experiencia presenta varios puntos a tener en cuenta, que a mi parecer son relevantes ya que son generadores de soluciones en la acción:

Identificación de una problemática ambiental alrededor de la cual se organiza a la comunidad educativa estudiantes, padres y maestros con la comunidad gubernamental y demás entidades relacionadas con la educación y la protección del ambiente; conformando una comunidad académica y un comité que posibilita y garantiza la marcha, eficacia y cubrimiento del proyecto.

A partir de la identificación de una serie de problemáticas ambientales diagnosticadas: la contaminación por residuos sólidos y aguas servidas del humedal, la falta de una conducta ciudadana frente a la conservación y manejo adecuado del humedal y la actitud negativa frente a las relaciones existentes con el entorno se inicia un proceso de creación de estrategias educativas, éticas, comportamentales y de concientización que comprometen a toda la comuni- dad a través de una apropiación del humedal no sólo como espacio biológico "ecosistema", sino como espacio geográfico, social, lúdico, investigativo, explorativo, artístico, que propicia el desarrollo de la creatividad y la apropiación de un conocimiento vivenciado.

La creación de un currículo acorde con las necesidades y expectativas comunitarias que permite la interacción de diversas entidades y que demuestra que los PEIs, no son estáticos, teóricos, inmodificables; por el contrario se alimentan de las problemáticas educativas, sociales, políticas, culturales y ambientales para ser elaborados.

La experiencia permite ver la necesidad de las instituciones educativas de liderar procesos de investigación y educación que se proyecten hacia diversas instancias como Redes, ONGs, grupos de investigación y universidades para que conjuntamente se potencien e impulsen dichos procesos.

Finalmente la experiencia deja la agradable sensación del trabajo conjunto, del amor, la apropiación y la identidad por lo que se tiene, por lo que se puede mejorar y por la existencia de millones de posibilidades de salvar nuestro planeta del gran predador, devastador y contaminador "el hombre".

\section{Denise Wanda Díaz Camelo}

Licenciada en Biología

Universidad Pedagógica Nacional

karalawan@hotmail.com 